\title{
DNA Evidence for Strong Genome-Wide Pleiotropy of Cognitive and Learning Abilities
}

\author{
Maciej Trzaskowski • Oliver S. P. Davis • \\ John C. DeFries $\cdot$ Jian Yang $\cdot$ Peter M. Visscher \\ Robert Plomin
}

Received: 13 February 2013/Accepted: 10 April 2013/Published online: 23 April 2013

(C) The Author(s) 2013. This article is published with open access at Springerlink.com

\begin{abstract}
Very different neurocognitive processes appear to be involved in cognitive abilities such as verbal and non-verbal ability as compared to learning abilities taught in schools such as reading and mathematics. However, twin studies that compare similarity for monozygotic and dizygotic twins suggest that the same genes are largely responsible for genetic influence on these diverse aspects of cognitive function. It is now possible to test this evidence for strong pleiotropy using DNA alone from samples of unrelated individuals. Here we used this new method with 1.7 million DNA markers for a sample of 2,500 unrelated children at age 12 to investigate for the first time the extent of pleiotropy between general cognitive ability (aka intelligence) and learning abilities (reading, mathematics and language skills). We also compared these DNA results to results from twin analyses using the same sample and measures. The DNA-based method revealed strong genome-wide pleiotropy: Genetic correlations were greater
\end{abstract}

Edited by John K Hewitt.

M. Trzaskowski $(\bowtie) \cdot$ O. S. P. Davis · R. Plomin

MRC Social, Genetic \& Developmental Psychiatry Centre, Institute of Psychiatry, King's College London, DeCrespigny Park, Denmark Hill, London SE5 8AF, UK

e-mail: maciej.trzaskowski@kcl.ac.uk

J. C. DeFries

University of Colorado, Institute for Behavioral Genetics, Boulder, CO 80309, USA

J. Yang · P. M. Visscher

University of Queensland Diamantina Institute, Princess

Alexandra Hospital, The University of Queensland, Brisbane,

QLD 4102, Australia

P. M. Visscher

Queensland Brain Institute, The University of Queensland,

Brisbane, QLD 4072, Australia than 0.70 between general cognitive ability and language, reading, and mathematics, results that were highly similar to twin study estimates of genetic correlations. These results indicate that genes related to diverse neurocognitive processes have general rather than specific effects.

Keywords Pleiotropy - Intelligence · Learning abilities · Mathematics · Language - GCTA · Twins · Heritability · Cognition

\section{Introduction}

Very different neurocognitive processes appear to be involved in cognitive abilities such as reasoning and mathematics (Deary 2000) However, quantitative genetic research, largely based on twin studies, consistently indicates that genes that affect individual differences in performance in one domain are largely the same genes that affect performance in other domains, leading to the Generalist Genes Hypothesis (Plomin and Kovas 2005).

It is now possible to use DNA itself to estimate genetic influence in any sample of unrelated individuals rather than relying on comparisons between monozygotic and dizygotic twins. The method, implemented in a tool called Genome-wide Complex Trait Analysis (GCTA; Yang et al. 2011a) does not attempt to identify specific genes associated with traits. Instead, it correlates genomic similarity across hundreds of thousands of single nucleotide polymorphisms (SNPs) with phenotypic similarity in a large sample of unrelated individuals (Yang et al. 2010). This population-based approach does not rely on the strong assumptions made in classical twin studies.

Univariate Linear Mixed Model (LMM) implemented in the GCTA package has been used to estimate genetic 
influence for height and body mass index (Yang et al. 2010, 2011b), psychiatric and medical disorders (Lee et al. 2011), personality (Vinkhuyzen et al. 2012), and cognitive abilities (Davies et al. 2011; Plomin et al. 2013b). In contrast to univariate genetic analysis, bivariate genetic analysis focuses on the genetic correlation, the correlation between genetic influences on different traits, called pleiotropy (Plomin et al. 2013a). High genetic correlations between phenotypes are often interpreted as an indication that the same genes affect the phenotypes. Genetic correlations between diverse cognitive abilities as estimated through twin studies are typically greater than 0.60 , indicating that cognition-related genes largely have general pleiotropic effects (Calvin et al. 2012; Plomin and Kovas 2005). However, the genetic correlation estimated from twin studies could be biased due to misspecification of the model of twin similarity for genetic and non-genetic effects. In this study, we use the GCTA package to estimate the genetic correlation between traits in conventionally unrelated individuals based on DNA evidence alone; this estimate is free of bias if we assume that the sole reason for phenotypic similarity between conventionally unrelated individuals is shared additive genetic factors. For brevity, we refer to LMM used in the GCTA package simply as GCTA.

Here we use bivariate GCTA (Lee et al. 2012; Yang et al. 2011a) to test the Generalist Genes Hypothesis by estimating genetic correlations between general cognitive ability (' $\mathrm{g}$ ', aka intelligence) and language, reading, and mathematics. We compare these genetic correlation estimates from GCTA to those obtained from the twin design using the same sample assessed at the same age with the same measures. We also analyze the variables of height and weight for purposes of comparison.

\section{Materials and methods}

Sample and genotyping

The sample was drawn from the Twins Early Development Study (TEDS), which is a multivariate longitudinal study that recruited over 11,000 twin pairs born in England and Wales in 1994, 1995 and 1996 (Haworth et al. 2012; Oliver and Plomin 2007). TEDS has been shown to be representative of the UK population (Kovas et al. 2007). The project received approval from the Institute of Psychiatry ethics committee (05/Q0706/228) and parental consent was obtained prior to data collection.

Cognitive and DNA data were available for 3,747 11and 12-year-old children whose first language was English and had no major medical or psychiatric problems. From that sample, 3,665 DNA samples were successfully hybridized to Affymetrix GeneChip 6.0 SNP genotyping arrays using standard experimental protocols as part of the WTCCC2 project (for details see Trzaskowski et al. 2013). In addition to nearly 700,000 genotyped SNPs, more than one million other SNPs were imputed from HapMap 2, 3 and WTCCC controls using IMPUTE v. 2 software (Howie et al. 2009). 3,152 DNA samples (1,446 males and 1,706 females) survived quality control criteria for ancestry, heterozygosity, relatedness, and hybridization intensity outliers. To control for ancestral stratification, we performed principal component analyses on a subset of 100,000 quality-controlled SNPs after removing SNPs in linkage disequilibrium $\left(\mathrm{r}^{2}>0.2\right)$ (Fellay et al. 2007). Using the Tracy-Widom test (Patterson et al. 2006), we identified 8 axes with $p<0.05$, which were used as covariates in GCTA analyses.

The mean age of the sample was 11.5 years $(\mathrm{SD}=0.66)$. The sample sizes for the GCTA results shown in Table 1 are 2,325 for ' $\mathrm{g}$ ' and language, 2,238 for ' $\mathrm{g}$ ' and mathematics, 2,250 for ' $\mathrm{g}$ ' and reading, and 2,296 for height and weight. For the twin analyses, cognitive data were available for 5,434 twin pairs (Davis et al. 2009); however, the twin analyses presented here were based only on twins included in the GCTA analyses in order to provide a more precise comparison between GCTA and twin-study results. The numbers of twin pairs were 2,205, 2,095, 2,104 and 2,162, respectively.

\section{Measures}

Cognitive data were collected online via the Internet using, where possible, adaptive branching, which enabled measurement of the full range of ability using a relatively small number of items. Details about the following measures, including references, are available elsewhere (Kovas et al. 2007).

\section{General cognitive ability $(g)$}

'g' was assessed from two verbal tests and two non-verbal tests. The verbal tests included WISC-III-PI Multiple Choice Information (General Knowledge) and Vocabulary

Table 1 Genome-wide Complex Trait Analysis (GCTA) and twin study estimates of genetic correlations. Standard errors (SE) are shown in parentheses. 'g' refers to general cognitive ability

\begin{tabular}{lrr}
\hline & \multicolumn{2}{c}{ Genetic correlation } \\
\cline { 2 - 3 } Bivariate comparison & GCTA (SE) & \multicolumn{1}{c}{ Twin (SE) } \\
\hline 'g' vs language & $0.81(0.15)$ & $0.80(0.06)$ \\
'g' vs mathematics & $0.74(0.15)$ & $0.73(0.03)$ \\
'g' vs reading & $0.89(0.26)$ & $0.66(0.05)$ \\
'g' vs height & $-0.13(0.30)$ & $-0.03(0.06)$ \\
'g' vs weight & $-0.04(0.25)$ & $-0.06(0.06)$ \\
Height vs weight & $0.76(0.13)$ & $0.65(0.02)$ \\
\hline
\end{tabular}


Multiple Choice subtest. The two non-verbal reasoning tests were WISC-III-UK Picture Completion and Raven's Standard and Advanced Progressive Matrices.

\section{Language}

Three components of language were assessed: syntax, semantics and pragmatics. Syntax was measured using the Listening Grammar subtest of the Test of Adolescent and Adult Language. Semantics was assessed using Level 2 of the Figurative Language subtest of the Test of Language Competence. Pragmatics was assessed using Level 2 of the Making Inferences subtest of the Test of Language Competence.

\section{Mathematics}

Assessment of mathematics targeted three components of mathematics: Understanding Number, Non-numerical Processes, and Computation and Knowledge. The items for these three scales were based on the National Foundation of Educational Research 5-14 Mathematics Series.

\section{Reading}

Four measures of reading were employed. Two measures assessed reading comprehension: the reading comprehension subtest of the Peabody Individual Achievement Test and the GOAL Formative Assessment in Literacy for Key Stage 3. Reading fluency was assessed by an adaptation of the Woodcock-Johnson III Reading Fluency Test and by the Test of Word Reading Efficiency, which was administered by telephone.

Composite measures for ' $\mathrm{g}$ ', language, mathematics, and reading. For each cognitive measure, outliers above or below $3 \mathrm{SD}$ from the mean were excluded. Scores were regressed on sex and age, and standardized residuals were derived and quantile normalized (Lehmann 1975; van der Waerden 1975). Composite measures for ' $\mathrm{g}$ ', language, mathematics, and reading were created as unit-weighted means requiring complete data for at least 3 of the 4 tests for ' $\mathrm{g}$ ' and reading and 2 of 3 tests for language and mathematics. All procedures were executed using R (www.r-project.org; R Development Core Team 2011). The phenotypic correlations among the composite measures were 0.63 for ' $\mathrm{g}$ ' and language, 0.63 for ' $\mathrm{g}$ ' and mathematics, and $0.57 \mathrm{for}$ ' $\mathrm{g}$ ' and reading.

\section{Height and weight}

Height and weight were assessed on the same sample (age 12) via self-report. Similar to the cognitive measures, outliers $( \pm 3 \mathrm{SD})$ were removed and scores were controlled for age and sex. The phenotypic correlation between height and weight was 0.63 .
Statistical analyses

GCTA

Conceptually, the amount of phenotypic variance, or covariance, explained by genetic factors is estimated by a comparison of a matrix of pairwise genomic similarity to a matrix of pairwise phenotypic similarity (Yang et al. 2010). Before the variance or covariance can be decomposed into genetic and residual components, we need to calculate pairwise genomic similarity between all pairs of individuals in the sample using all genetic markers genotyped on the SNP array. Because the GCTA package uses a random effects model to estimate genetic effects from a sample of unrelated individuals in the population, any pair whose genetic similarity is equal to or greater than a fourth cousin is removed (estimate of pairwise relatedness $>0.025$ ). In univariate analysis, the variance of a trait can be partitioned using residual maximum likelihood into genetic and residual components. Detailed description of this method can be found in GCTA publications (Yang et al. 2010, 2011a, b). The bivariate method extends the univariate model by relating the pairwise genetic similarity matrix to a phenotypic covariance matrix between traits 1 and 2 (Lee et al. 2012). The eight principal components described earlier were used as covariates in our bivariate GCTA analyses; as mentioned in the previous section, all phenotypes were age- and sexregressed prior to analysis.

Twin modelling. The twin design and model-fitting is discussed elsewhere (Plomin et al. 2013a). We fit a bivariate Cholesky decomposition using OpenMx (Boker et al. 2011), which provided a direct comparison with the bivariate GCTA. The correlated factor solution is the least restricted model allowing variables to correlate with one another via genetic, shared environment, and non-shared environment. Because previous analyses of these data indicated nonsignificant differences in model-fitting results between males and females (Kovas et al. 2007), we combined same-sex and opposite-sex DZ twin pairs in order to increase the power of the analyses.

\section{Results}

Table 1 shows GCTA-estimated genetic correlations (and standard errors, SE) between ' $\mathrm{g}$ ' and learning abilities for more than 2,238 12-year-old UK twins (randomly selecting only one member of each twin pair to control for potential confounds, such as birth order) based on 1.7 million SNPs measured from the Affymetrix 6.0 GeneChip or imputed from HapMap 2,3 and WTCCC controls (Trzaskowski et al. 2013). Genetic correlations are significant and 


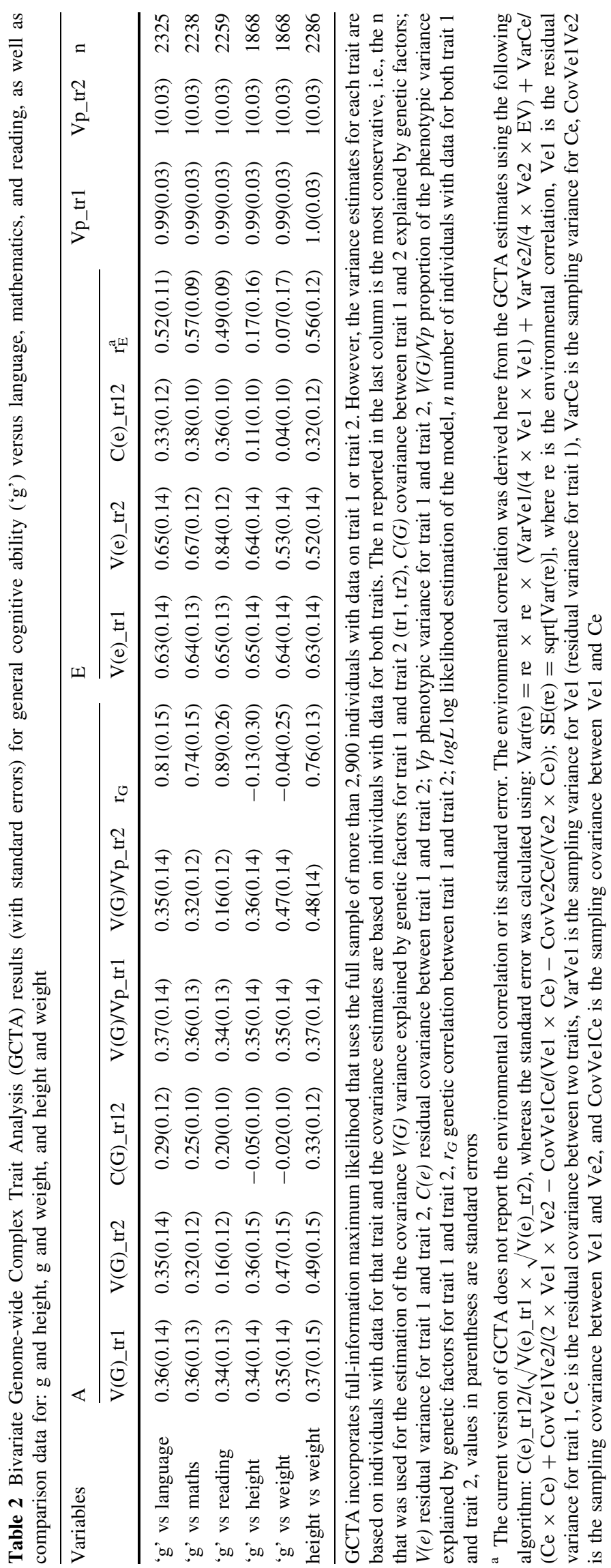




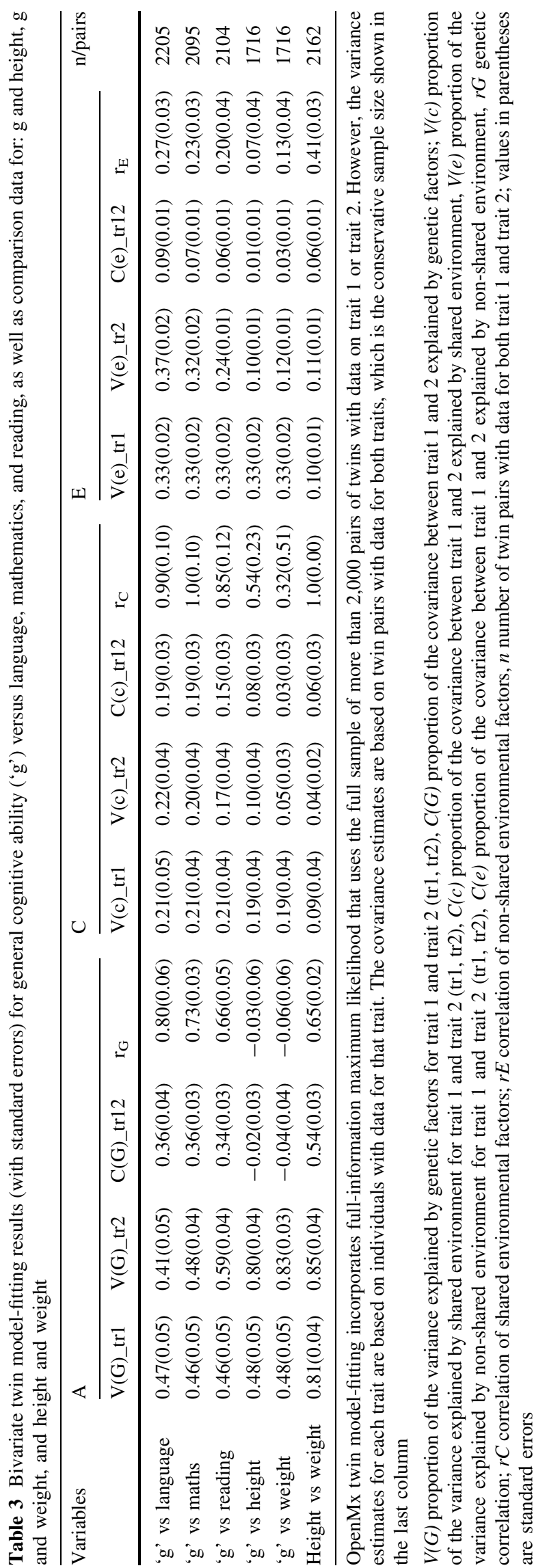

substantial for all three comparisons-between ' $\mathrm{g}$ ' and language (0.81), mathematics (0.74), and reading (0.89). The GCTA-estimated genetic correlations between ' $\mathrm{g}$ ' and learning abilities are similar in magnitude to the GCTAestimated genetic correlation between height and weight (0.76). In addition, Table 1 includes bivariate results for ' $g$ ' versus height and ' $g$ ' versus weight as 'negative controls'; their phenotypic correlations are both 0.07. As expected, these comparisons yielded negligible and nonsignificant genetic correlations $(-0.03$ and -0.06 , respectively).

Table 1 also includes analogous genetic correlations from twin model-fitting analyses, as estimated from the same twin sample but including the co-twins (more than 2,095 pairs of twins). The GCTA-estimated genetic correlations are highly similar to the twin study estimates and do not differ significantly, as indicated by their overlapping standard errors. The similarity of GCTA and twin estimates of genetic correlations extend to the comparison between height and weight as well as the negative control comparisons of ' $\mathrm{g}$ ' and height and ' $\mathrm{g}$ ' and weight.

Tables 2 and 3 show full results from the bivariate GCTA and twin analyses, respectively.

\section{Discussion}

Using DNA evidence alone, these high genetic correlations estimated from GCTA support the Generalist Genes Hypothesis in showing strong pleiotropy between ' $\mathrm{g}$ ' and learning abilities, especially because we show that these GCTA-estimated genetic correlations are as high as genetic correlations estimated from the twin design.

Although GCTA does not identify specific genes associated with these traits, it addresses a critical issue in genomewide association studies: the extent to which common SNPs used on commercially available SNP arrays can account for the heritability of quantitative traits (Yang et al. 2011b). We have shown in univariate GCTA analyses that, if samples were sufficiently large, common SNPs could account for more than two-thirds of the heritability of cognitive abilities estimated in twin studies (Yang et al. 2011b; see also Table 2). Why are univariate GCTA heritability estimates less than the twin study estimates of heritability? As discussed elsewhere (e.g. Yang et al. 2010), the main problem is imperfect tagging. The common SNPs used on all available commercial arrays only capture what is in LD with them. Rare variants, which have lower minor allele frequency, will thus not be 'tagged' and their influence will be missed. In addition, GCTA estimates additive genetic influence only, so that non-additive effects (gene-gene and gene-environment interaction) are not captured either.

A more novel question, and central to the present paper, is why, as we have shown here, bivariate genetic 
correlations estimated by GCTA are as great as twin study estimates. The likely reason is that attenuation of the estimated additive genetic variance due to imperfect linkage disequilibrium between causal variants and genotyped SNPs applies to both the additive genetic variance of the two traits and to their additive genetic covariance by the same proportion. Thus, the GCTA estimate of the genetic correlation is unbiased because it is derived from the ratio between genetic covariance and the genetic variances of the two traits.

Are generalist genes all in the mind (cognition) or are they in the brain as well? That is, genetic correlations between cognitive and learning abilities might be epiphenomenal in the sense that multiple genetically independent brain mechanisms could affect each ability, creating genetic correlations among abilities. However, the genetic principles of pleiotropy (each gene affects many traits) and polygenicity (many genes affect each trait) lead us to predict that generalist genes have their effects further upstream, creating genetic correlations among brain structures and functions, a prediction that supports a network view of brain structure and function.

Acknowledgments Twins Early Development Study (TEDS) is supported by a program grant to RP from the UK Medical Research Council (G0901245, and previously G0500079), with additional support from the US National Institutes of Health (HD044454; HD046167). Genome-wide genotyping was made possible by a grant from the Wellcome Trust to the Wellcome Trust Case Control Consortium 2 project (085475/B/08/Z; 085475/Z/08/Z). RP is supported by a Medical Research Council Research Professorship award (G19/ 2) and a European Advanced Investigator award (295366); MT is supported by a Medical Research Council studentship; OSPD is supported by a Sir Henry Wellcome Fellowship from the Wellcome Trust (WT088984). The authors declare that they had no conflicts of interest with respect to their authorship or publication of this article.

Open Access This article is distributed under the terms of the Creative Commons Attribution License which permits any use, distribution, and reproduction in any medium, provided the original author(s) and the source are credited.

\section{References}

Boker S, Neale M, Maes H, Wilde M, Spiegel M, Brick T, Spies J, Estabrook R, Kenny S, Bates T, Mehta P, Fox J (2011) OpenMx: an open source extended structural equation modeling framework. Psychometrika 76(2):306-317

Calvin C, Deary I, Webbink D, Smith P, Fernandes C, Lee S, Luciano M, Visscher P (2012) Multivariate genetic analyses of cognition and academic achievement from two population samples of 174,000 and 166,000 school children. Behav Genet 42(5):699-710

Davies G, Tenesa A, Payton A, Yang J, Harris SE, Liewald D, Ke X, Le Hellard S, Christoforou A, Luciano M, McGhee K, Lopez L, Gow AJ, Corley J, Redmond P, Fox HC, Haggarty P, Whalley LJ, McNeill G, Goddard ME, Espeseth T, Lundervold AJ,
Reinvang I, Pickles A, Steen VM, Ollier W, Porteous DJ, Horan M, Starr JM, Pendleton N, Visscher PM, Deary IJ (2011) Genome-wide association studies establish that human intelligence is highly heritable and polygenic. Mol Psychiatry 16(10):996-1005

Davis OSP, Haworth CMA, Plomin R (2009) Learning abilities and disabilities: generalist genes in early adolescence. Cog Neuropsychiatry 14(4-5):14312-14331

Deary I (2000) Looking down on human intelligence: from psychometrics to the brain. Oxford University Press, Oxford

Fellay J, Shianna KV, Ge D, Colombo S, Ledergerber B, Weale M, Zhang K, Gumbs C, Castagna A, Cossarizza A, Cozzi-Lepri A, De Luca A, Easterbrook P, Francioli P, Mallal S, MartinezPicado J, Miro JM, Obel N, Smith JP, Wyniger J, Descombes P, Antonarakis SE, Letvin NL, McMichael AJ, Haynes BF, Telenti A, Goldstein DB (2007) A whole-genome association study of major determinants for host control of HIV-1. Science 317(5840):944-947

Haworth CMA, Davis OSP, Plomin R (2012) Twins Early Development Study (TEDS): a genetically sensitive investigation of cognitive and behavioural development from childhood to young adulthood. Twin Res Hum Genet 16(1):117-125

Howie BN, Donnelly P, Marchini J (2009) A flexible and accurate genotype imputation method for the next generation of genomewide association studies. PLoS Genet 5(6):e1000529

Kovas Y, Haworth CMA, Dale PS, Plomin R (2007) The genetic and environmental origins of learning abilities and disabilities in the early school years. Monogrs Soc Res Child Dev 72(3):144-721

Lee SH, Wray NR, Goddard ME, Visscher PM (2011) Estimating missing heritability for disease from genome-wide association studies. Am J Hum Genet 88(3):294-305

Lee SH, Yang J, Goddard ME, Visscher PM, Wray NR (2012) Estimation of pleiotropy between complex diseases using SNPderived genomic relationships and restricted maximum likelihood. Bioinformatics 28(19):2540-2542

Lehmann EL (1975) Nonparametrics: statistical methods based on ranks. Holden-Day, San Francisco

Oliver BR, Plomin R (2007) Twins Early Development Study (TEDS): A multivariate, longitudinal genetic investigation of language, cognition and behaviour problems from childhood through adolescence. Twin Res Human Genet 10(1):96-105

Patterson N, Price AL, Reich D (2006) Population structure and eigenanalysis. PLoS Genet 2(12):2074-2093

Plomin R, Kovas Y (2005) Generalist genes and learning disabilities. Psychol Bull 131:592-617

Plomin R, DeFries JC, Knopik VS, Neiderhiser JM (2013a) Behavioral genetics. Worth Publishers, New York

Plomin R, Haworth CMA, Meaburn EL, Price TS, Wellcome Trust Case Control Consortium 2, Davis OSP (2013b) Common DNA markers can account for more than half of the genetic influence on cognitive abilities. Psychol Sci 24(4):562-568

R Development Core Team (2011) R: a Language and environment for statistical computing. Vienna, Austria

Trzaskowski M, Eley TC, Davis OSP, Doherty SJ, Hanscombe KB, Meaburn EL, Haworth CMA, Price T, Plomin R (2013) First genome-wide association study on anxiety-related behaviours in childhood. PLoS One 8(4):e58676

van der Waerden BL (1975) On the sources of my book "Moderne Algebra". Historia Mathematica 2:31-40

Vinkhuyzen AA, Pedersen NL, Yang J, Lee SH, Magnusson PK, Iacono WG, McGue M, Madden PA, Heath AC, Luciano M, Payton A, Horan M, Ollier W, Pendleton N, Deary IJ, Montgomery GW, Martin NG, Visscher PM, Wray NR (2012) Common SNPs explain some of the variation in the personality dimensions of neuroticism and extraversion. Transl Psychiatry 2:e102 
Yang J, Benyamin B, McEvoy BP, Gordon SD, Henders AK, Nyholt DR, Madden PA, Heath AC, Martin NG, Montgomery GW, Goddard ME, Visscher PM (2010) Common SNPs explain a large proportion of the heritability for human height. Nat Genet 42(7):565-569

Yang JA, Lee SH, Goddard ME, Visscher PM (2011a) GCTA: a tool for genome-wide complex trait analysis. Am J Hum Genet $88(1): 76-82$
Yang J, Manolio TA, Pasquale LR, Boerwinkle E, Caporaso N, Cunningham JM, De Andrade M, Feenstra B, Feingold E, Hayes MG (2011b) Genome partitioning of genetic variation for complex traits using common SNPs. Nat Genet 43(6):519-525 